Wolfgang E. Nagel · Willi Jäger · Michael Resch Editors

\title{
High Performance Computing in Science and Engineering '06
}

Transactions of the High Performance Computing Center Stuttgart (HLRS) 2006

With 312 Figures, 101 in Colour, and 46 Tables 


\section{Contents}

\section{Physics}

H. Ruder and R. Speith ............................ 1

Gravitational Wave Signals from Simulations of Black Hole Dynamics

B. Brügmann, J. Gonzalez, M. Hannam, S. Husa, P. Marronetti,

U. Sperhake, and W. Tichy ........................... 3

The SuperN-Project: Understanding Core Collapse Supernovae

A. Marek, K. Kifonidis, H.-Th. Janka, and B. Müller ............ 19

MHD Code Optimizations and Jets in Dense Gaseous Halos

V. Gaibler, M. Vigelius, M. Krause, and M. Camenzind .......... 35

Anomalous Water Optical Absorption:

Large-Scale First-Principles Simulations

W.G. Schmidt, S. Blankenburg, S. Wippermann, A. Hermann,

P.H. Hahn, M. Preuss, K. Seino, and F. Bechstedt............. 49

The Electronic Structures of Nanosystems:

Calculating the Ground States of Sodium Nanoclusters

and the Actuation of Carbon Nanotubes

B. Huber, L. Pastewka, P. Koskinen, M. Moseler ................ 59

Object-Oriented SPH-Simulations with Surface Tension

S. Ganzenmüller, A. Nagel, S. Holtwick, W. Rosenstiel, and H. Ruder . 69

Simulations of Particle Suspensions at the Institute

for Computational Physics

J. Harting, M. Hecht, and H. Herrmann .................. 83 
VIII Contents

\section{Solid State Physics}

W. Hanke.

Nano-Systems in External Fields and Reduced Geometry:

Numerical Investigations

P. Henseler, C. Schieback, K. Franzrahe, F. Bürzle, M. Dreher,

J. Neder, W. Quester, M. Kläui, U. Rüdiger, and P. Nielaba ........ 97

Signal Transport and Finite Bias Conductance

in and Through Correlated Nanostructures

P. Schmitteckert and G. Schneider .....................113

Atomistic Simulations of Dislocation - Crack Interaction

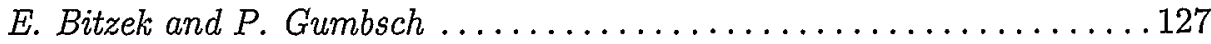

Monte Carlo Simulations of Strongly Correlated

and Frustrated Quantum Systems

C. Lavalle, S.R. Manmana, S. Wessel, and A. Muramatsu

Chemistry

C. van Wüllen

Characterization of Catalyst Surfaces by STM Image Calculations

R. Kovacik, B. Meyer, and D. Marx.

Theoretical Investigation of the Self-Diffusion on $\mathrm{Au}(100)$

K. Pötting, T. Jacob, and W. Schmickler

TrpAQP: Computer Simulations to Determine

the Selectivity of Aquaporins

M. Dynowski and U. Ludewig.

\section{Computational Fluid Dynamics}

S. Wagner

Direct Numerical Simulation and Analysis of the Flow Field

Around a Swept Laminar Separation Bubble

T. Hetsch and U. Rist

Direct Numerical Simulation of Primary Breakup Phenomena

in Liquid Sheets

W. Sander and B. Weigand

Direct Numerical Simulation of Mixing and Chemical Reactions in a Round Jet into a Crossflow - a Benchmark

J.A. Denev, J. Fröhlich, and H. Bockhorn

Numerical Simulation of the Bursting

$O$. Marxen and D. Henningson 
Parallel Large Eddy Simulation with UG

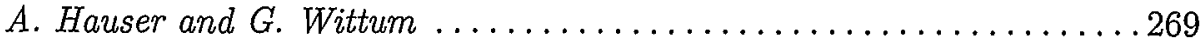

LES and DNS of Melt Flow and Heat Transfer

in Czochralski Crystal Growth

A. Raufeisen, M. Brewer, V. Kumar, T. Botsch, and F. Durst.......279

Efficient Implementation of Nonlinear Deconvolution Methods

for Implicit Large-Eddy Simulation

S. Hickel and N.A. Adams .............................. 293

Large-Eddy Simulation of Tundish Flow

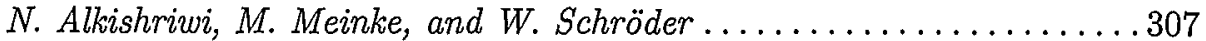

Large Eddy Simulation of Open-Channel Flow Over Spheres

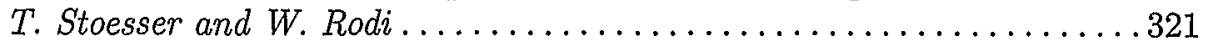

Prediction of the Resonance Characteristics of Combustion Chambers on the Basis of Large-Eddy Simulation

F. Magagnato, B. Pritz, H. Büchner, and M. Gabi ...............331

Investigations of Flow and Species Transport in Packed Beds

by Lattice Boltzmann Simulations

T. Zeiser ...................................... 343

Rheological Properties of Binary

and Ternary Amphiphilic Fluid Mixtures

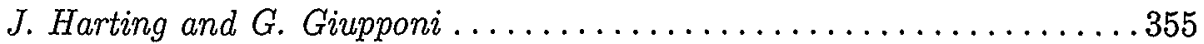

The Effects of Vortex Generator Arrays on Heat Transfer

and Flow Field

C.F. Dietz, M. Henze, S.O. Neumann, J. von Wolfersdorf,

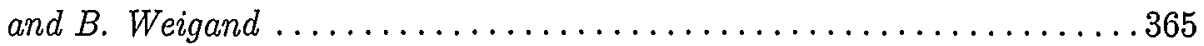

Investigation of the Influence of the Inlet Geometry

on the Flow in a Swirl Burner

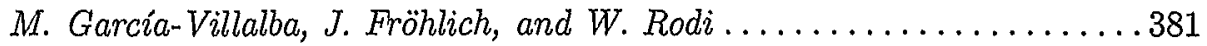

Numerical Investigation and Simulation of Transition Effects

in Hypersonic Intake Flows

M. Krause, B. Reinartz, and J. Ballmann ..............................

Aeroelastic Simulations of Isolated Rotors Using Weak

Fluid-Structure Coupling

M. Dietz, M. Kessler, and E. Krämer ...................407

Computational Study of the Aeroelastic Equilibrium Configuration of a Swept Wind Tunnel Wing Model in Subsonic Flow

L. Reimer, C. Braun, and J. Ballmann ...................421 


\section{Structural Mechanics}

P. Wriggers .................................... 435

Numerical Prediction of the Residual Stress State after Shot Peening M. Klemenz, M. Zimmermann, V. Schulze, and D. Löhe...........4437

Computer-Aided Destruction of Complex Structures by Blasting

S. Mattern, G. Blankenhorn, and K. Schweizerhof................ 449

Wave Propagation in Automotive Structures Induced by Impact Events

S. Mattern and K. Schweizerhof ......................... 459

\section{Miscellaneous Topics}

W. Schröder

Continental Growth and Thermal Convection in the Earth's Mantle

$U$. Walzer, R. Hendel, and J. Baumgardner .................473

Efficient Satellite Based Geopotential Recovery

O. Baur, G. Austen, and W. Keller

Molecular Modeling of Hydrogen Bonding Fluids: Monomethylamine, Dimethylamine, and Water Revised

T. Schnabel, J. Vrabec, and H. Hasse...

The Application of a Black-Box Solver with Error Estimate to Different Systems of PDEs

T. Adolph and W. Schönauer.............................5527

Scalable Parallel Suffix Array Construction

$F$. Kulla and P. Sanders. 